\title{
INSTITUTOS E LABORATÓRIOS PRIVADOS DE PESQUISA: UMA PROPOSTA DE CONCEITUAÇÃO E DELINEAMENTO COMO UM AGENTE DO SISTEMA NACIONAL DE INOVAÇÃO SOB A ÓTICA NO NOVO MARCO LEGAL DA INOVAÇÃO
}

\author{
Welbert Luiz Silva, Saulo Henrique Vidigal Maciel ${ }^{2}$ e Raoni Barros Bagno ${ }^{3}$ \\ ${ }^{1}$ NTQI-UFMG, welbert-luiz@ufmg.br \\ ${ }^{2}$ NTQI-UFMG, saulohvm@gmail.com \\ ${ }^{3}$ NTQI-UFMG, rbagno@dep.ufmg.br
}

\section{RESUMO}

A proposta desse trabalho é conceituar Institutos e Laboratórios Privados de Pesquisa (ILPP) e delinear a participação como um agente do Sistema Nacional de Inovação (SNI). Estudos preliminares compõem uma vasta bibliografia que conceitua e descreve a participação de Universidades e Institutos de Pesquisa como agentes do SNI. A maior parte desses estudos detalham claramente as experiências e práticas da interação entre estes agentes, dando-lhes papel central em detrimento das contribuições de outros agentes. Assim, este estudo procura avaliar a contribuição potencial dos ILPP junto às empresas para o desenvolvimento de inovações. Dessa forma, a proposta desse trabalho é: i) conceituar e caracterizar o termo ILPP; ii) analisar a importância dos ILPP segundo dados da PINTEC; iii) apresentar uma amostra dos Institutos e Laboratórios Privados de Pesquisa; iv) comparar o grupo amostral com a definição de ICT da Lei $n^{\circ} 13.243$ (BRASIL, 2016 art. $2^{\circ}$ ). Dessa forma esse trabalho pretende abrir um campo de pesquisa ainda pouco explorado no ambiente teórico, porém, já em aplicação no ambiente empresarial.

Palavras-chaves: Inovação, Sistema Nacional de Inovação, Institutos e Laboratórios Privados de Pesquisa.

\begin{abstract}
This paper aims to conceptualize Private Research Institutes (PRI) and describe its participation as an agent of the National Innovation System (SNI). This study evaluates the PRI's contribution to the development of innovations. In conclusion, the main focus of this research is: i) conceptualize and characterize the term PRI; ii) analyze the importance of PRI; iii) present a sample of Private Research Institutes; iv) and make a comparison between the sample and the Brazilian Innovation Law (Law 13.243, Brasil, 2016).
\end{abstract}

Keywords: Innovation, National Innovation System, Private Research Institutes.

Área 11. Empreendedorismo, redes, arranjos produtivos e inovação.

Classificação JEL: O17 Formal and Informal Sectors, Shadow Economy, Institutional Arrangements 
Ao analisarmos o processo de industrialização brasileiro, verifica-se a dependência histórica da extração das riquezas naturais e a produção de commodities agrícolas e, por outro lado, a dependência da expansão dos negócios das multinacionais que aqui se instalaram através de subsidiárias (FLEURY, 1999). Nesse contexto, o Brasil se especializou na exportação de produtos de baixo valor agregado e na importação de tecnologia. Isso vem representando, nas últimas décadas, um grande atraso produtivo e econômico se comparado aos países mais avançados. (RIBEIRO et al., 2006)

Schumpeter (1911) que define inovação como sendo a introdução no mercado de um bem novo, um novo método de produção, a abertura de novos mercados, a conquista de novas fontes de oferta de matériaprima, o estabelecimento de um novo negócio. Dessa forma, o investimento em inovação é a chave para o domínio do conhecimento em sua essência, know-why, e, em contrapartida, é um caminho seguro para retirar o país da dependência da exploração de recursos naturais e da importação de tecnologias. Pelo investimento em inovação torna-se possível deter o domínio das tecnologias que associadas a geração de produtos de maior valor agregado dão suporte ao desenvolvimento econômico do país.

Porém, para gerar inovação as empresas enfrentam e são impactadas por fatores econômicos, sociais, políticos, organizacionais, institucionais e entre outros. Tal contexto é capturado pelo conceito de Sistema de Inovação (SI) conforme descrito do Edquist (2006). Para o autor a abordagem de SI agrupa as perspectivas nacional, setorial e regional discutidas por: Carlsson (1995) sistemas tecnológicos; Breschi e Malerba (1997) sistema setorial; e Braczyk et. al. (1998), Cooke (2001) e Asheim e Isaksen (2002) sistema regional de inovação.

A expressão Sistema Nacional de Inovação - SNI - foi publicada a primeira vez por Freeman (1987). Atualmente Lundvall (1992) e Nelson (1993) são duas referências importantes para o debate de SNI. Nelson enfatiza estudo de casos empíricos, mais pesadamente, que a teoria desenvolvida e alguns dos estudos estritamente do sistema nacional de P\&D. Em contraste, Lundvall busca desenvolver uma alternativa à tradicional economia neoclássica para aprendizagem nos locais de interação, tendo a inovação com centros de análise. Para Lundvall, a "estrutura de produção e o arranjo institucional" são as duas mais importantes dimensões que definem o SI. Nelson (1993) foca as organizações que promovem, criam e disseminam o conhecimento como a principal fonte de inovação.

Nesse sentido as Universidades e os Institutos de Ciências e Tecnologia (ICTs) desempenham um papel fundamental no SNI. Assim, a proposta desse trabalho é conceituar Institutos e Laboratórios Privados de Pesquisa (ILPP) no campo das ICTs e delinear a participação dos ILPP como um agente do Sistema Nacional de Inovação (SNI).

Dessa forma, esse trabalho apresenta: i) o conceito e a caracterização do termo ILPP; ii) uma análise da importância dos ILPP segundo dados da PINTEC; iii) uma amostra dos Institutos e Laboratórios Privados de Pesquisa; iv) a comparação do grupo amostral com a definição de ICT da Lei no 13.243 (BRASIL, 2016 art. 2o). Em síntese, o trabalho de pesquisa pretende responder as seguintes questões: $1^{\text {a }}$. Qual a importância dos ILPP para o desenvolvimento da inovação no país? 2a . Quais são e onde estão localizados os ILPP? $3^{\mathrm{a}}$. Que tipos de atividades são realizadas por esses institutos? 4 ${ }^{\mathrm{a}}$. Quantos atendem a definição de ICT da atual legislação? 5a. Quais são as competências necessárias de um ILPP para a execução das atividades de ciência e tecnologia?

Nesse artigo, apresenta-se os levantamentos preliminares que buscam responder a os questionamentos 1 e 2 do trabalho de pesquisa em andamento.

\section{ARCABOUÇO LEGAL E NORMATIVO}

Analisando o arcabouço legal da inovação no Brasil, a Lei No 10.973 (Brasil, 2004 art. 2º definiu ICT como sendo: 
Instituição Científica e Tecnológica - ICT: órgão ou entidade da administração pública que tenha por missão institucional, dentre outras, executar atividades de pesquisa básica ou aplicada de caráter científico ou tecnológico; (Grifo nosso)

Conforme a Lei, apenas órgãos ou entidades da administração pública eram considerados como ICT. Assim, deixava de fora, da abrangência da lei, os institutos privados.

O atual Marco Regulatório da Inovação corrige essa falha ao descrever no texto da Lei $\mathrm{n}^{0} 13.243$ (BRASIL, 2016, art. $2^{\circ}$ ) ICT como sendo:

Instituição Científica, Tecnológica e de Inovação (ICT): órgão ou entidade da administração pública direta ou indireta ou pessoa jurídica de direito privado sem fins lucrativos legalmente constituída sob as leis brasileiras, com sede e foro no País, que inclua em sua missão institucional ou em seu objetivo social ou estatutário a pesquisa básica ou aplicada de caráter científico ou tecnológico ou o desenvolvimento de novos produtos, serviços ou processos; (Grifo nosso)

Dessa forma, o atual Marco Regulatório da Inovação abrange, no escopo do conceito de ICT os institutos de ciência e tecnologia de direito privado e sem fins lucrativos, abrindo, foco para um grupo de organizações ainda pouco exploradas no escopo do SNI.

Ao delinear a abrangência de escopo das Atividades de Ciências e Tecnologia o Manual de Frascati (OECD, 2002) descreve que:

(...) As atividades de ciência e tecnologia incluem, além de $P \& D$, educação e formação científicas e tecnológicas (CTET), além de serviços científicos e técnicos (STC). Estes últimos englobam, por exemplo, serviços de C\&T prestados por bibliotecas e museus, a tradução e a publicação de obras sobre C\&T, o levantamento topográfico e a prospecção, coleta de informação sobre fenômenos socioeconômicos, os testes, a padronização e o controle de qualidade, as atividades de consultoria para os clientes bem como as atividades sobre patentes e licenças por parte do governo e administrações públicas. (Grifo nosso)

Ao definir os limites da atividade de P\&D do Manual de Frascati (OECD, 2002) descreve que:

As instituições ou unidades da instituição e as empresas onde P\&D é a principal atividade $e$ que têm frequentemente atividades secundárias outras além de $P \& D$ (informação científica e técnica, teste, controle de qualidade, análise etc.). Na medida em que a atividade secundária fosse principalmente realizada nos interesses de $P \& D$, ela deveria ser classificada como $P \& D$. Se ela destina-se principalmente a satisfazer a necessidades outras que aquelas de $P \& D$, esta deveria ser excluída. (Grifo nosso)

Em síntese, pode-se definir que as atividades de teste, controle da qualidade e análise se enquadram como atividade de $P \& D$ quando realizadas nos interesses da pesquisa e desenvolvimento. Por outro lado, podese considerar como Atividade de Ciência e Tecnologia, os testes, a padronização e o controle de qualidade, as atividades de consultoria para os clientes quando prestados na forma de Serviços Científicos e Técnicos. Sob essa ótica, esse conceito traz para o campo das ICT toda a matriz de instituições e laboratórios que prestam serviços, desde a realização de testes até o desenvolvimento de pesquisas.

Assim, pode-se considerar Institutos e Laboratórios Privados de Pesquisa (ILPP) como uma parte das instituições que compõem o conceito de ICT atualmente descrito na lei. Ou seja, no âmbito do conceito de ICT, os ILPP não abrangem as universidades e os centros de formação profissional.

Por outro lado, ainda sob o foco do conceito de ICT, os ILPP não se limitam a instituições sem fins lucrativos bem como também não excluem as instituições que não possuam em sua missão institucional 
ou em seu objetivo social ou estatutário as atividades de P\&D. Sendo que, a inclusão na missão institucional a atividade de P\&D é uma análise estratégica que os institutos devem realizar para acessarem as prerrogativas da lei.

Dessa forma, pode-se delinear como ILPP os Institutos e Laboratórios Privados de Pesquisa que tenham, ou não, fins lucrativos, e que desenvolvam atividades de: teste, controle de qualidade, análise, atividades de consultoria, pesquisa e desenvolvimento.

3.

O PAPEL DOS ILPP NO SNI

Tidd et. al., (2008), sob a ótica da Gestão da Inovação, descrevem que para as empresas executarem suas estratégias de inovação não podem ignorar o sistema nacional de inovação em que estão inseridas. Os autores destacam que os sistemas nacionais de inovação abrem oportunidades ou impõem restrições às empresas. Esses sistemas influenciam as demandas e as condições competitivas, as ofertas de recursos humanos e a regulamentação da iniciativa privada.

No Brasil, a Pesquisa de Inovação Tecnológica - PINTEC, é uma pesquisa sobre os fatores que influenciam o comportamento inovador das empresas, sobre as estratégias adotadas, os esforços empreendidos, os incentivos, os obstáculos e os resultados da inovação.

A Pesquisa de Inovação PINTEC - 2011 (IBGE, 2012) avalia 15 (quinze) temas relacionados à inovação. Desses temas, dois merecem destaque para analisar a importância dos ILPP no comportamento inovador das empresas, são eles: i) Fonte de Informação: nesse bloco de questões, são identificadas as fontes de ideias e de informações utilizadas no processo de inovação; ii) Relações de cooperação para inovação: são questões que avaliam a participação ativa da empresa em projetos conjuntos de P\&D e outros projetos de inovação com outra organização (empresa ou instituição), o que não implica, necessariamente, que as partes envolvidas obtenham benefícios comerciais imediatos.

Com base nos dados a PINTEC 2011 (IBGE, 2013), no período 2009-2011, com um universo de 128699 empresas com 10 ou mais pessoas ocupadas. Desse total, 116632 são empresas do setor das Indústrias extrativas e de transformação, 503 empresas são do setor de Eletricidade e Gás e 11564 são empresas do setor de Serviços. A tabela 1 apresenta a participação de empresas por setor de abrangência da PINTEC.

Tabela 1 - Participação de empresas que implementaram inovação por setor de abrangência da PINTEC

\begin{tabular}{|c|c|c|c|c|}
\hline \multirow[b]{3}{*}{$\begin{array}{l}\text { Variáveis selecionadas das empresas nas } \\
\text { atividades da indústria, do setor de ele- } \\
\text { tricidade e gás e dos serviços seleciona- } \\
\text { dos - Brasil - período 2009-2011 }\end{array}$} & \multicolumn{4}{|c|}{ 2009-2011 } \\
\hline & & \multicolumn{3}{|c|}{ Que implementaram } \\
\hline & & $\begin{array}{l}\text { Inovação } \\
\text { De } \\
\text { Produto } \\
\text { e/ou } \\
\text { Processo } \\
\end{array}$ & $\begin{array}{c}\text { Apenas } \\
\text { projetos } \\
\text { incompletos } \\
\text { e/ou aban- } \\
\text { donados } \\
\end{array}$ & $\begin{array}{c}\text { Apenas } \\
\text { inovações } \\
\text { organizacio- } \\
\text { nais e/ou de } \\
\text { marketing } \\
\end{array}$ \\
\hline Total & 128699 & 45950 & 3158 & 44955 \\
\hline Indústrias extrativas e de transformação & 116632 & 41470 & 2743 & 41312 \\
\hline Eletricidade e Gás & 503 & 222 & 60 & 128 \\
\hline Serviços & 11564 & 4258 & 354 & 3516 \\
\hline
\end{tabular}

Fonte: IBGE, Diretoria de Pesquisas, Coordenação de Indústria, Pesquisa de Inovação 2011.

Na pesquisa PINTEC (Gráfico 1), acima mencionada, é avaliado o grau de importância das seguintes fontes externas de informação para gerar inovação.

Gráfico 1 - Empresas das indústrias extrativa e de transformação que implementaram inovações, por grau de importância alto das fontes de informação empregada 


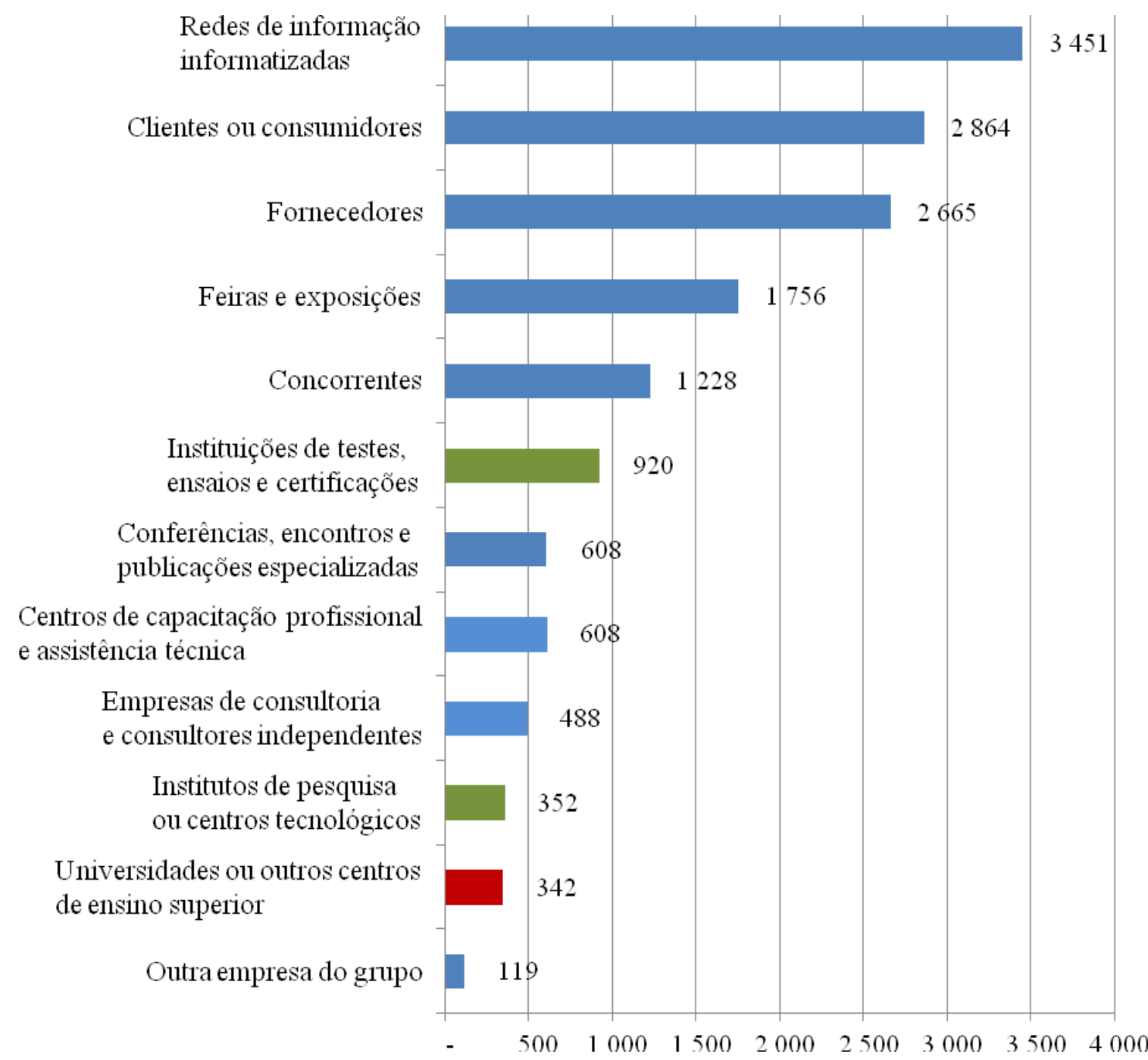

Fonte: IBGE, Diretoria de Pesquisas, Coordenação de Indústria, Pesquisa de Inovação 2011.

Das 41470 indústrias extrativas e de transformação que implementaram inovação de produto e/ou processo, 920 avaliam que institutos de testes, ensaios e certificações são uma fonte de alta importância para gerar inovação e 352 avaliam os institutos de pesquisa ou centros tecnológicos como sendo uma fonte de alta importância. Se considerarmos que os ILPP são tanto a Institutos de teste quanto os centros tecnológicos, a avaliação de importância alta da fonte de informação será um número próximo a avaliação de importância alta da fonte de informação de "concorrentes". Isso pode ser um indicativo do grau de importância do ILPP como gerador de informação para o SNI.

Com relação à cooperação das empresas como outros atores para gerar inovação de produto ou processo, a PINTEC avalia a importância alta, média e baixa da cooperação. O gráfico 2 apresenta a importância de interação.

Gráfico 2 - Empresas das indústrias extrativa e de transformação que implementaram inovações, por grau de importância alta da cooperação com outras organizações 


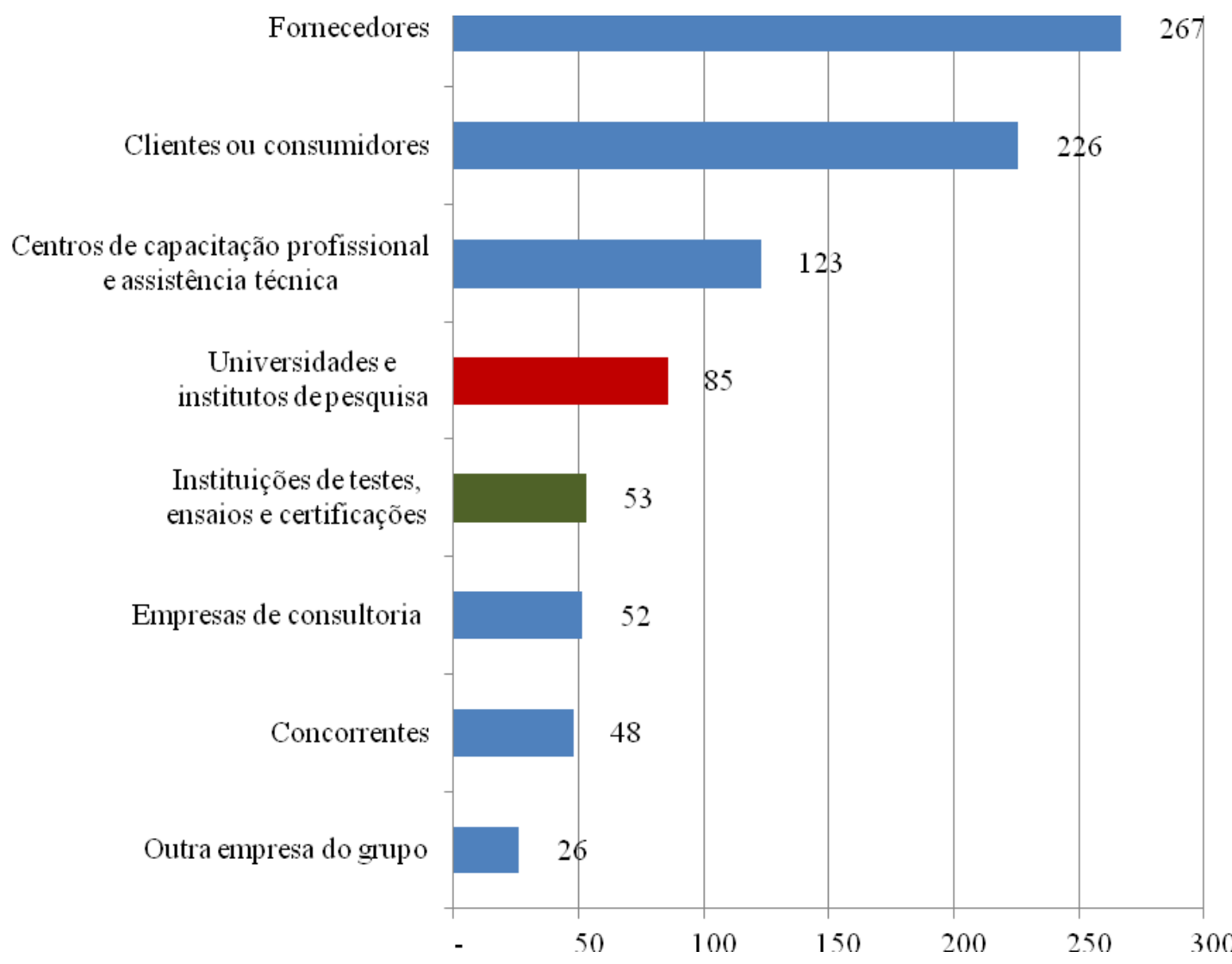

Fonte: IBGE, Diretoria de Pesquisas, Coordenação de Indústria, Pesquisa de Inovação 2011.

Ao avaliar a importância da cooperação pode-se verificar que a pesquisa considera na avaliação, Universidades e Institutos de Pesquisa como um mesmo grupo, isso interfere na análise. Outro fator que também deve ser destacado nessa análise é o escopo de prestação de serviço dos institutos de teste, o que dificulta a percepção de oportunidades de parcerias para cooperação.

Em síntese, pela análise das informações da PINTEC 2011 (IBGE, 2013), pode-se observar que os Institutos e Laboratórios Privados de Pesquisa (ILPP) apresentam uma importância relevante sob o aspecto da fonte de informação, porém, como cooperação na geração de inovação, esses institutos ainda necessitam de uma maior disseminação de oportunidades.

4.

METODOLOGIA DE MAPEAMENTO DOS ILPP NO

BRASIL

Para o mapeamento dos Institutos e Laboratórios Privados de Pesquisa foram utilizadas duas fontes de informação. Primeiro foram levantadas informações disponíveis na base de dados RAIS (Relação Anual de Informações Sociais) do Ministério do Trabalho e Previdência Social - MTPS. A segunda fonte de informação foram as listas de laboratórios acreditados do INMETRO referentes à: RBC Rede Brasileira de Calibração, RBLE Rede Brasileira de Laboratórios de Ensaio, PEP Provedores de Ensaios de Proficiência, PMR Produtores de Material de Referência e OCP Organismos de Certificação de Produto.

A base de dados RAIS é um levantamento anual em que constam os dados das empresas públicas ou privadas com a Classificação Nacional de Atividades Econômicas - CNAE. Para as análises foram utilizados os dados da RAIS 2014 cadastrados com os CNAE 7120100: Testes e Análises Técnicas e CNAE 7210000: Pesquisa e Desenvolvimento Experimental em Ciências Físicas e Naturais. 
Na base de dados do INMETRO há todos os laboratórios acreditados além de informações importantes referentes ao escopo do serviço prestado por esses laboratórios. O cruzamento das duas fontes de informação teve como objetivo: $1^{\circ}$. inserir na fonte de dados da RAIS as informações técnicas disponíveis na base de dados do INMETRO; $2^{\circ}$. identificar laboratórios importantes da matriz laboratorial cadastrados na base de Dados RAIS com um código de CNAE diferente dos selecionados.

Na base de dados RAIS 2014 foram executadas as seguintes etapas de análise de dados: $1^{\circ}$. Foram listadas todas as instituições e laboratórios cadastrados com os CNAES (7120100 e 7210000) primário ou secundário; $2^{\circ}$. Foram excluídas instituições e laboratórios com 0 (zero) funcionários; $3^{\circ}$. Foram excluídos as instituições e laboratórios cadastrados como Órgão da Administração Pública; $4^{\circ}$. Foram excluídos os registros de laboratório de análise clínica, laboratórios de certificação veicular e laboratório de análise ambiental; $5^{\circ}$. Foram excluídos os registros de instituições e laboratórios com 1, 2 ou 3 funcionários sem site ou informação na internet; $6^{\circ}$. Foram excluídas os institutos e laboratórios caracterizados como empresas de capital misto.

Na primeira etapa da coleta de dados da RAIS 2014 foram encontrados 5010 registros. Após a aplicação dos filtros obteve-se uma lista com 977 instituições e laboratórios.

Na base de dados do INMETRO, as informações foram coletadas em 28/11/2015 das redes laboratoriais selecionadas e realizadas as seguintes etapas de análise de dados: $1^{\circ}$. Foram listados todos os laboratórios acreditados pelo INMETRO da: RBC Rede Brasileira de Calibração, RBLE Rede Brasileira de Laboratórios de Ensaio, PEP Provedores de Ensaios de Proficiência, PMR Produtores de Material de Referência e OCP Organismos de Certificação de Produto; $2^{\circ}$. Foi elaborada uma lista única com todas as instituições e laboratórios; $3^{\circ}$. Foi feita a fusão dos dados em uma única linha para cada laboratório e retirada de duplicação de dados; $4^{\circ}$. Foram excluídos os laboratórios de análise clínica, de laboratórios de certificação veicular e laboratório de análise ambiental; $5^{\circ}$. Foram excluídas os institutos e laboratórios caracterizados como empresa públicas e de capital misto.

A lista única com todos os laboratórios acreditados do INMETRO constava 1230 registros. Após a aplicação dos filtros obteve-se uma lista com 989 registros.

Após esses levantamentos, foi elaborada uma lista única com os dados tratados originados da RAIS 2014 e do site do INMETRO. Para a elaboração dessa lista foram executadas as seguintes etapas: $1^{\circ}$. Foi elaborada uma lista única RAIS e INMETRO; $2^{\circ}$. Foi retirada a duplicação e feita a fusão dos dados em uma única linha para cada instituição ou laboratório.

Na etapa anterior, inicialmente havia 1966 registros. Após a retirada das duplicações e fusão de dados, obteve-se uma lista com 1887 instituições e laboratórios. Desse total, $898 \mathrm{com}$ dados apenas de origem da RAIS 2014 (CNAE 7120100 e CNAE 7210000 ), 910 são instituições ou laboratórios de origem apenas de informações da lista do INMETRO e 79 possuem informações de ambas as listas.

5.

\section{RESULTADOS E DISCUSSÃO}

A lista final com os 1887 registros é uma amostra representativa dos institutos de pesquisa e laboratórios de testes que podem se encaixar no conceito de ILPP definido nesse artigo. O gráfico 3 apresenta a distribuição por Região e Estado dos Institutos e Laboratórios Privados de Pesquisa. 
Gráfico 3 - Distribuição de Institutos e Laboratórios Privados de Pesquisa por Região e Estado

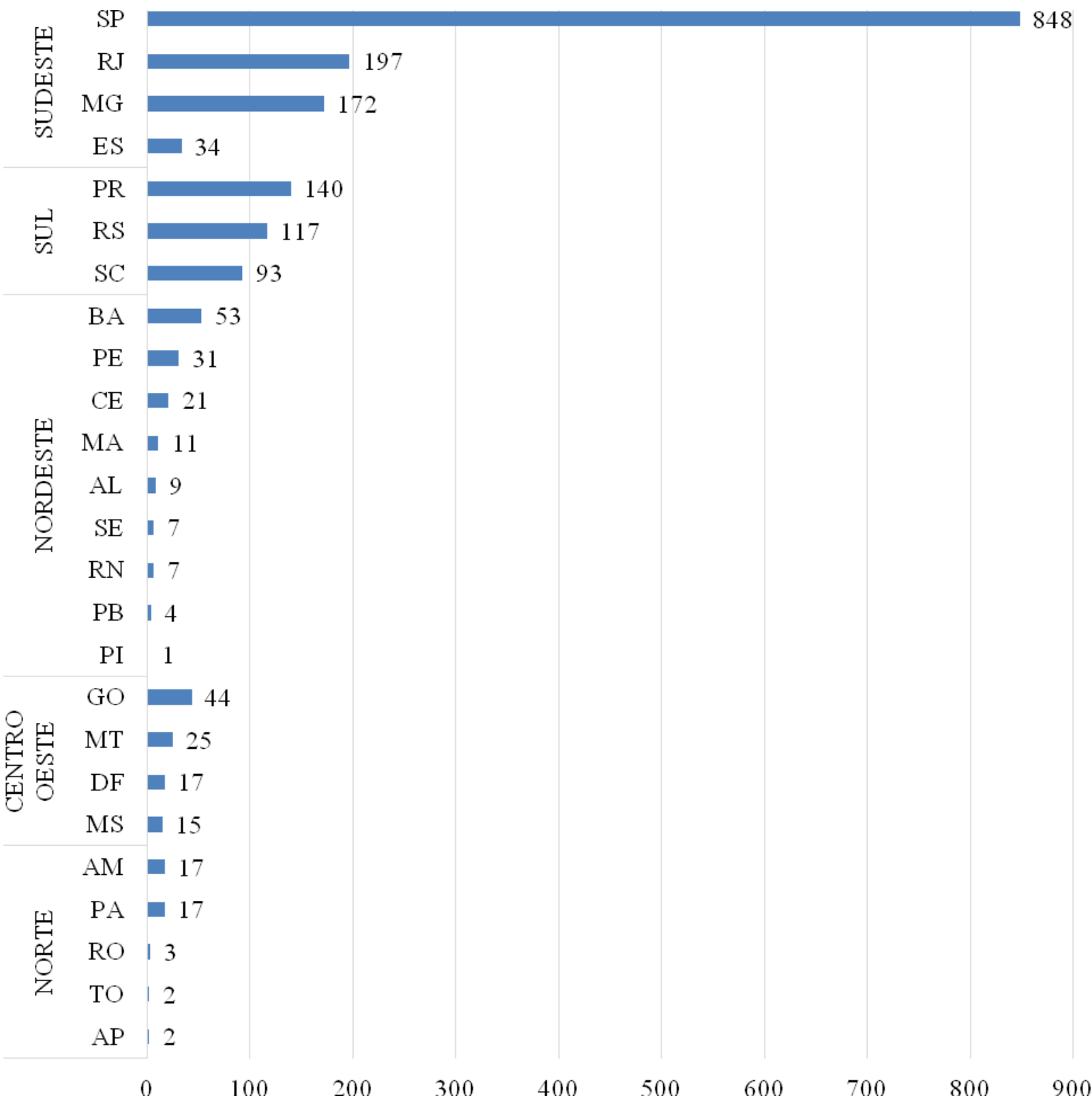

Fonte: Elaborado pelos autores utilizando como base de dados a RAIS 2014 e o site do INMETRO

Conforme apresentado, o Gráfico 3 é uma tentativa de quantificar, a partir de dados disponíveis, o que seria a distribuição dos ILPP no território nacional. Em relação ao conceito, a amostra apresenta as seguintes limitações: primeiro, essa amostra não consegue capturar informações de institutos ou laboratórios que não foram cadastrados com os CNAES selecionados e que não possuem acreditação no INMETRO. Nesse caso, há uma dificuldade de levantar informações pela falta de um identificador ou rede que os agrupe.

A segunda limitação é que na amostra pode haver instituto ou laboratório que desenvolva algum tipo de ensaio que não se encaixe nas atividades de Ciência e Tecnologia descritas no Manual de Frascati.

Os dados da amostra retratam informações importantes como a concentração de institutos e laboratórios no estado de São Paulo e na Região Sudeste do país e a ausência de registros nos estados do Acre e Roraima da região Norte do Brasil. 
Ao analisarmos a participação dos maiores grupos de institutos e laboratórios a amostra apresenta distribuição conforme gráfico 4.

Gráfico 4 - Maiores Grupos de Institutos e Laboratórios de Amostra

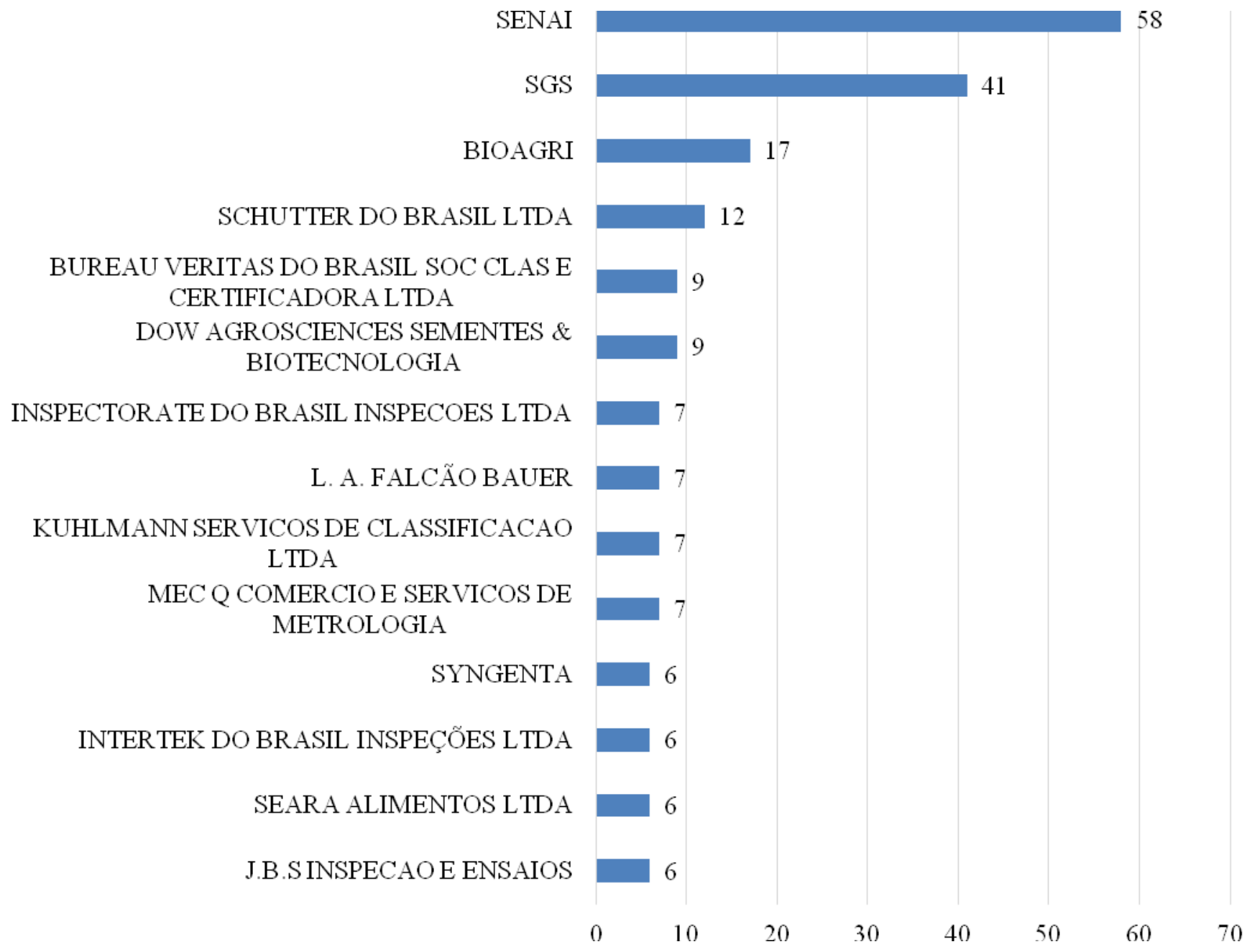

Fonte: Elaborado pelos autores utilizando como base de dados a RAIS 2014 e o site do INMETRO

O gráfico apresenta os grupos de institutos e laboratórios com até 6 unidades diferentes. Os maiores grupos de institutos e laboratórios correspondem a 198 registros (10\% da amostra).

Sob a ótica das certificações a tabela 2 apresenta a quantidade de institutos e laboratórios privados de pesquisa por tipo de certificação do INMETRO.

Tabela 2 - Distribuição de Certificações do INMETRO da Amostra

\begin{tabular}{l|r}
\hline \multicolumn{1}{c|}{ CERTIFICAÇÕES } & QUANTIDADE \\
\hline SEM CERTIFICAÇÃO & 898 \\
RBLE REDE BRASILEIRA DE LABORATÓRIOS DE ENSAIO & 637 \\
RBC REDE BRASILEIRA DE CALIBRAÇÃO & 323 \\
OCP ORGANISMOS DE CERTIFICAÇÃO DE PRODUTO & 72 \\
PEP PROVEDORES DE ENSAIOS DE PROFICIÊNCIA & 12 \\
PMR PRODUTORES DE MATERIAL DE REFERÊNCIA & 4 \\
\hline
\end{tabular}

Fonte: Elaborado pelos autores utilizando como base de dados a RAIS 2014 e o site do INMETRO 
Pela análise desses dados pode-se observar que 898 institutos ou laboratórios privados de pesquisa (48\% do total) não apresentam nenhum tipo de certificação ou acreditação do INMETRO.

6.

\section{CONCLUSÃO}

A despeito da dificuldade de mapeamento e caracterização dos Institutos e Laboratórios Privados de Pesquisa (ILPP), os dados levantados nesse trabalho apresentam informações importantes sobre a matriz de institutos e laboratorial de apoio a inovação no país.

Quando analisado a distribuição dos ILPP no Brasil, verifica-se a forte incidência na região Sudeste e principalmente uma grande concentração no estado de São Paulo. Esse dado é compatível com o estudo organizado por De Negri \& Squeff (2016) que demonstra a conentração de infraestrutura de inovação e pesquisa na região Sudeste do país.

Se analisada o tipo de serviço prestados pelos ILPP da área industrial, pode-se observar que as atividades são muito mais orientadas para avaliação da conformidade (inspeção, ensaios, calibrações e certificação) do que para pesquisa, desenvolvimento e extensão industrial.

Sob a ótica das certificações, observa-se que aproximadamente a metade dos institutos e laboratórios que compõem a amostra, não apresentação certificações ou acreditações das redes de laboratórios do INMETRO.

Segundo o conceito de ICT do novo Marco Legal da Inovação, os ILPP sem fins lucrativos que queiram se caracterizar como ICT e que: i) possuem certificação, podem utilizar o seu sistema de gestão para demonstrar que a sua missão institucional é orientado para a pesquisa; ii) são cadastrados com CNAE 7120100 ou CNAE 7210000, podem utilizar os dados cadastrais para demostrar que seu objetivo social ou estatutário está ligado à pesquisa; iii) são cadastrados com CNAE 7120100 ou CNAE 7210000 e que possuam alguma certificação, poderão utilizar as duas formas para demostrar que desenvolvem atividades de pesquisa; iv) não são cadastrados com CNAE 7120100 ou CNAE 7210000 ou que não tenham alguma certificação, deverão se adaptar ou utilizar outras formas para atender à especificação da lei.

A próxima atividade do trabalho de pesquisa é levantar que tipos de atividades são realizadas pelos institutos ou laboratórios que compõem a amostra. A partir desse levantamento, agrupar em níveis de atividades de inovação os institutos ou laboratórios que: i) são dedicados a realizar ensaios e teste; ii) ofertam consultorias ou assistência técnica; iii) desenvolvem pesquisa em parceria; iv) fazem transferência de tecnologia desenvolvida internamente.

Para cada um dos níveis de atividades em inovação, descrever uma matriz de competências necessárias para que os institutos possam evoluir nos níveis de prestação de serviços, utilizando como referencial comparativo a norma ABNT NBR 16501 que descreve diretrizes para sistemas de gestão da pesquisa e da inovação (PD\&I) e o Modelo Descritivo da Capacidade Tecnológica proposto por Figueiredo (2005).

Apesar de ser uma análise introdutória, esse debate abre uma série de oportunidades, tanto na área acadêmica quanto de estratégias de mercado, para acadêmicos e gestores de institutos ou laboratórios.

\section{REFERÊNCIAS}

ASSOCIAÇÃO BRASILEIRA DE NORMAS TÉCNICAS. NBR 16501: Diretrizes para sistemas de gestão da pesquisa e da inovação (PD\&I). Rio de Janeiro, 2011.

ASHEIM, B., and ISAKSEN, A. Regional Innovation Systems: The Integration of Local 'Sticky' and Global 'Ubiquitous' Knowledge, Journal of Technology Transfer 27: 77-86, 2002. 
BRASIL. Lei $\mathbf{n}^{0} \mathbf{1 0 . 9 7 3}$ de 2 de dezembro de 2004. Dispõe sobre incentivos à inovação e à pesquisa científica e tecnológica no ambiente produtivo e dá outras providências.

BRASIL. Lei $\mathbf{n}^{0}$ 13.243de 11 de janeiro de 2016. Dispõe sobre estímulos ao desenvolvimento científico, à pesquisa, à capacitação científica e tecnológica e à inovação e dá outras providências.

BRACZYK, H.-J., COOKE, P., e HEIDENREICH, M. (eds.), Regional Innovation Systems: The Role of Governance in a Globalised World, London and Pennsylvania: UCL, 1998.

BRESCHI, S., e MALERBA, F., Sectoral Innovation Systems: Technological Regimes, Schumpeterian Dynamics, and Spatial Boundaries, in Edquist, 1997a: 130-56. 1997

CARLSSON, B. (ed.), Technological Systems and Economic Performance: The Case of Factory Automation, Dordrecht. Kluwer.Study, 1995.

COOKE, P., Regional Innovation Systems, Clusters, and the Knowledge Economy, Industrial and Corporate Change 10(4): 945-74, 2001.

DE NEGRI, F. (Org.); SQUEFF, F. H. S. (Org.), Sistemas setoriais de inovação e infraestrutura de pesquisa no Brasil. Brasília : IPEA : FINEP : CNPq, 2016.

EDQUIST, C., Systems of Innovation: Perspectives and ChallengesThe Oxford Handbook of Innovation. Oxford, 2006.

FIGUEIREDO, P. N., Acumulação tecnológica e inovação industrial: conceitos, mensuração e evidências no Brasil. São Paulo em Perspectiva, v. 19, n. 1, p. 54-69, 2005.

FLEURY, A., Gerenciamento Do Desenvolvimento De Produtos.Business,1999.

FREEMAN, C., Technology Policy and Economic Performance: Lessons fron Japan, London: Printer. Find This Resource, 1987.

IBGE. Manual - Pesquisa de Inovação PINTEC - 2011. 2012.

IBGE. Pesquisa de Inovaçao - PINTEC. p. 227, 2013.

LUNDVALL, B -A. (ed.), Nacional Systems of Innovation: Towards a Theory of Innovation and Interactive Learning, London: Pinter. 1992.

NELSON, R. R. (ed.), National Systems of innovation: A Comparative Sudy. Oxford, Oxford University Press, 1993.

TIDD, J. BESSANT, J. PAVITT, K., Gestão da inovação. 3. Ed - Porto Alegre: Bookman, 2008.

OCDE, Manual de Frascati. p. 282, 2002.

RIBEIRO, L. RUIZ, R. BERNARDES, A. ALBUQUERQUE, E., Running Twice as Fast ?Methods, p. 81-87, 2006.

SCHUMPETER, J., A teoria do desenvolvimento econômico. Editora Nova Cultural Ltda.1985. 